\title{
Comorbid OCD, higher score on the MFQ, and older age at presentation predicted persistence of major depression in children and adolescents
}

\author{
Goodyer IM, Herbert J, Secher SM, et al. Short-term outcome of major depression: I. Comorbidity and severity at presentation as predictors \\ of persistent disorder.J Am Acad Child Adolesc Psychiatry 1997 Feb;36:179-87.
}

Goodyer IM, Herbert J, Tamplin A, et al. Short-term outcome of major depression: II. Life events, family dysfunction, and friendship difficulties as predictors of persistent disorder.J Am Acad Child Adolesc Psychiatry. 1997 Apr;36:474-80.

\section{Objective}

To determine whether comorbid disorders, duration of current episode, severity of depressive symptoms at presentation, long standing social difficulties, recent life events, current marital and family function, and friendship difficulties at presentation and 9 months later predict persistence of major depression at 36 weeks follow up.

\section{Design}

Inception cohort followed up for 36 weeks.

\section{Setting}

Child and adolescent mental health services in Cambridge, England.

\section{Patients}

78 patients (74 outpatients and 4 inpatients) between 8 and 16 years of age with a first episode of major depression diagnosed using the Diagnostic and Statistical Manual of Mental Disorders,
$3 r d$ edition, revised (DSM-III-R). 68 patients (87\%) were reassessed at 36 weeks.

\section{Assessment of prognostic factors}

At presentation and 36 weeks follow up, current mental state was assessed using the Schedule for Affective Disorders and Schizophrenia for School Age Children-Present episode version (K-SADS-P), and severity of current depression was determined using the self report version of the Mood and Feelings Questionnaire (MFQ). Mother and child completed the K-SADS-P separately with 2 interviewers. In addition, at presentation, mothers and children completed an extensive semistructured interview regarding background and psychosocial factors. At presentation and again at 36 weeks follow up, information was obtained from parents using interview and questionnaire procedures on current long term difficulties (current employ-

(continued on page 25)

\section{Commentary}

These 2 studies by Goodyer et al aim to identify clinical and social context predictors of the immediate clinical course of childhood MDD. Earlier work has established that children and adolescents frequently become depressed (2-7\% MDD prevalence rates in adolescents). ${ }^{1}$ The symptoms are generally the same as those experienced by depressed adults, comorbid internalising and externalising disorders are common, and while children often recover somewhat faster from an index episode than adults do, they have a higher recurrence rate into adulthood, and are thought to be at higher risk than depressed adults of developing bipolar disorder. ${ }^{2}$ While previous studies have assessed whether selected characteristics are predictors of clinical course and have documented various social cognitive correlates of childhood depression, none has looked as carefully in a longitudinal study at the influence of comorbid disorders, life events, and social contextual factors on clinical course.

The studies by Goodyer $e t$ al were well done. There was a low drop out rate (13\%), although it is not clear how many patients refused participation, and there were missing data for the family functioning measure. The short follow up period allowed for assessment of prediction of MDD recovery and persistence but not of MDD recurrence over the long term. Therefore, the findings are most relevant to immediate prognosis of MDD and to the development of acute treatments. The studies included sophisticated methods for measuring life events as well as good measures of psychopathology, peer relationships, and family functioning. No measures of depressogenic cognitions or of biological correlates of the depressive state (which are useful prognostic variables in studies of adult depression ${ }^{3}$ ) were reported in these 2 articles. However, 2 previously published papers provide these data. ${ }^{4} \mathrm{~A}$ methodological strength was the assessment of comorbid psychopathology at follow up and the inclusion of analyses to assess whether variables that predicted MDD persistence and recovery also predicted persistence of other psychiatric disorder. This feature allowed the investigators to assess whether identified predictors were specific to MDD outcomes.
The following findings will interest clinicians. Firstly, comorbid psychopathology was common and many patients had nondepressive disorders at 9 months follow up. The authors emphasise the need for clinicians to assess coexisting psychopathology in depressed children. It is also reasonable to conclude that comorbid disorders should be a focus of treatment. The identification of OCD as a predictor of MDD persistence is a new finding consistent with an earlier study which found that anxiety disorders predicted MDD course in adolescents. $^{6}$

Goodyer et al suggest that interpersonal or cognitive behavioural therapies could be considered for anxious depressed children. A logical next step would be to evaluate whether, as an adjunct to antidepressant therapy, treatments specifically targeting anxiety improve outcome. The high cooccurrence of MDD with conduct and oppositional defiant disorders (highest after separation anxiety disorder) points to the importance of untangling putative relationships among these overlapping disorders.

(continued on page 25) 
(abstract continued from page 24)

ment, quality of housing, and current income; number of people living in the house, number of children, and any child registered with social services; current marital status, confiding relationships with partner or another, and verbal or physical conflict between parents). Recent life events (classified into 3 psychological domains: danger, disappointment, and loss) and friendship difficulties were assessed at presentation and at 9 months follow up by semistructured interview. The mother and child completed the interview independently. Family function was measured using the Family Assessment Device (FAD).

\section{Main outcome measure}

$D S M-I I I-R$ diagnosis at 36 weeks. The main outcome groupings were depressed (major depressive disorder [MDD] and any other disorder), psychiatric disorder (no MDD but any other disorder), and recovered.

\section{Main results}

At presentation, 63 patients $(93 \%)$ had $\geqslant 1$ comorbid psychiatric disorder (median $=3)$ ). At 36 weeks, 17 patients $(25 \%\{95 \%$ CI $15 \%$ to $37 \%$; ) were classified as recovered, 17 (25\% \{CI 15\% to $37 \%\}^{*}$ ) continued to meet diagnostic criteria for $\geqslant 1$ psychiatric disorder but not major depression, and 34 (50\% \{CI 38\% to $62 \%$ *) still met criteria for DSM-III-R major depression (25 [73\% \{CI 56\% to 87\%\}*] had been persistently depressed and 9 [27\% \{CI 13\% to 44\%\}*] had recovered and subsequently relapsed). Major depression at follow up was predicted by 3 features at presentation; comorbid obsessive compulsive disorder (OCD) (adjusted odds ratio [OR] 5.5 \{CI 1.1 to 28.1 $\}^{*}$ ), age (OR for each year increase 1.5 \{CI 1.1 to 2.0$\}^{*}$ ), and higher MFQ score (OR for each point increase 1.1 \{CI 1.0 to 1.2$\left.\}^{*}\right)$. A model including comorbid oppositional defiant disorder at presentation (OR 27.8 \{CI 1.8 to 430.1 \}) in addition to the same 3 variables (ORs 8.9 \{CI 0.7 to 113.1 \}*, 1.5 \{CI 1.1 to 2.1$\}^{*}, 1.1$ \{CI 1.0 to 1.1$\}^{*}$, respectively), was predictive of persistent psychiatric disorder, and persistent depression was more likely in patients with a longer duration of illness before presentation. A model including 3 psychosocial factors were associated with persistent psychiatric disorder in general (but not with persistent major depression specifically); severely disappointing events between presentation and follow up (OR 18.9 \{CI 2.7 to 134.3\}*), lack of a maternal confiding relationship with current partner (OR 17.0 \{CI 1.4 to 208.3$\}^{*}$ ), and family dysfunction (OR 4.4 \{CI 0.7 to $\left.28.5\}^{*}\right)$ at presentation

\section{Conclusions}

Comorbid obsessive compulsive disorder, older age, and a higher score on the Mood and Feelings Questionnaire at presentation predicted persistence of major depression at 36 weeks follow up in patients with a first episode of major depression. A model including severely disappointing events between presentation and follow up, lack of a maternal confiding relationship with current partner, and family dysfunction at presentation predicted persistent psychiatric disorder in general, but not major depression specifically.

*Numbers calculated from data in article.

Source of funding: The Wellcome Trust.

For article reprint: Professor I M Goodyer, Developmental Psychiatry Section, Douglas House, $18 b$ Trumpington Road, Cambridge CB2 2AH, UK. Fax +44 (0)1223 324661. (commentary continued from page 24)

Oppositional defiant disorder was a predictor of the persistence of other psychiatric disorder but not of MDD specifically. Adjunctive treatments targeted at oppositional and conduct disorders should also be evaluated in depressed children.

Secondly, older children were at greater risk of more persistent disorder than younger children which is consistent with earlier findings that older adolescents are also at higher risk of adult mood disorder. ${ }^{7}$ Together, these findings provide a timely reminder that the general enthusiasm to focus treatment efforts early in childhood should be tempered with concern not to neglect treatment in mid to late adolescence.

Thirdly, more severe depressive symptoms predicted MDD persistence. In adults, severe depressive symptoms also identify patients who show poor response to cognitive behavioural therapy alone. ${ }^{3}$ The same may apply in childhood, although the issue needs further study. It would also be useful to evaluate whether residual symptoms beyond MDD remission predict MDD recurrence as this should have implications for treatment selection and timing of treatment withdrawal.

Fourthly, the most interesting new findings concern psychosocial contextual factors and life events. Lack of maternal confiding relationship with current partner and disappointing life events between presentation and follow up were predictive of persistent psychiatric disorder but not specifically of MDD persistence. These findings raise the possibility that contextual factors are not specific to disorder but constitute separate domains that influence the course of any disorder. Clearly, we cannot draw this conclusion from a study restricted to depressed children, but the relation between social context and psychopathology should be a focus of future work. In any event, clinicians should include reliable and valid measures of life events, social contextual factors, and impaired social functioning as these will provide useful information about subsequent course. Treatments targeted more specifically at these contexts and designed to buffer life stressors (eg, family psychoeducation, parent support, marital therapy, and peer group therapies) should be considered.
Again, it would be useful to evaluate these therapies as sole or adjunctive treatments in child and adolescent MDD.

\section{Mark Sanford, MBChB McMaster University Hamilton, Ontario, Canada}

1 Fleming JE, Offord DR. Epidemiology of childhood depressive disorders: a critical review. $J$ Am Acad Child Adolesc Psychiatry 1990;29:57180 .

2 Kovacs M. Presentation and course of major depressive disorder during childhood and
later years of the life span. I Am Acad Child later years of the life span. J Am
Adolesc Psychiatry 1996;35:705-15.

3 Sanford M, Szatmari P, Spinner M, et al. Predicting the one-year course of adolescent major depression. J Am Acad Child Adolesc Psychiatry 1995;34:1618-28.

4 Goodyer IM, Herbert J, Altham PM, et al. Adrenal secretion during major depression in 8- to 16-year-olds, I. Altered diurnal rhythms in salivary cortisol and dehydroepiandrosterone (DHEA) at presentation. Psychol Med 1996;26: 245-56.

5 Herbert J, Goodyer IM, Altham PM, et al. Adrenal secretion and major depression in 8- to 16 -year-olds, II. Influence of co-morbidity at 16-year-olds, II. Influence of co-morbidity a
presentation. Psychol Med 1996;26:257-63.

6 Harrington R, Fudge H, Rutter M, et al. Adult Harrington R, Fudge H, Rutter M, et al. Adult
outcomes of child and adolescent depression. outcomes of child and adolescent depression.
I. Psychiatric status. Arch Gen Psychiatry 1990; I. Psychiatric

7 Thase ME, Simons AD, Reynolds CF. Psychobiological correlates of poor response to cognitive behaviour therapy: potential indicators for antidepressant pharmacotherapy. Psychopharmacol Bull 1993;29:293-301. 